\title{
EPIDEMIOLOGY OF DRUG RESISTANT TUBERCULOSIS IN SAMTSE GENERAL HOSPITAL, BHUTAN: A RETROSPECTIVE STUDY
}

\section{Dorji $\mathrm{T}^{1}$}

${ }^{1}$ Ministry of Health, Samtse General Hospital, Bhutan

\begin{abstract}
Introduction: Multidrug resistant tuberculosis (MDR-TB) is defined as a case of tuberculosis resistant to rifampicin and isoniazid which are the first line anti tuberculosis drugs. Globally emergences of MDR-TB possess a challenge to TB control. In Bhutan, the proportion of MDR-TB is high at par with the global level. This study will explore the predictors of MDR-TB and the trend at Samtse General Hospital which has high burden of tuberculosis.
\end{abstract}

Methods: This was a retrospective cross sectional study. The data was extracted from TB treatment cards maintained at TB unit of Samtse General Hospital TB from January 2012 to June 2018.

Results: The study showed the prevalence of drug resistant to at least one drug at $21 \%$ and MDR-TB prevalence at $16 \%$. The patients with previous treatments (AOR: $4.59 ; 95 \% \mathrm{Cl} .03-18$ ) and patients under the age of 30 years (AOR: $2.7 ; 95 \% \mathrm{Cl} 1.01-7.42$ ) were significantly associated with MDR-TB.

Conclusion: This study shows high prevalence of MDR-TB in Samtse. Better strategies and concrete actions need to be developed to combat the increase of MDR-TB.

Key words: MDR, Gene Xpert,Primary MDR-TB, drug resistant, TB, Bhutan

\section{INTRODUCTION}

Tuberculosis (TB) is an infectious disease caused by Mycobacterium tuberculosis and is one of the top ten killers in the world second after HIV (human immunodeficiency virus). ${ }^{1}$

Commendable success has been made in reducing the TB burden through implementation of Directly Observed Treatment Short course (DOTS). However, the fight against TB has been hampered by emergence of multi-drug resistant tuberculosis (MDR-TB) and HIV. ${ }^{2}$ MDR-TB is defined as type of mycobacterium tuberculosis strain that is resistant to the two main first line anti tuberculosis drugs - rifampicin and isoniazid. ${ }^{3}$ MDR-TB has been declared as public health problem by World Health Organisation (WHO) in 2013.MDR-TB similar to

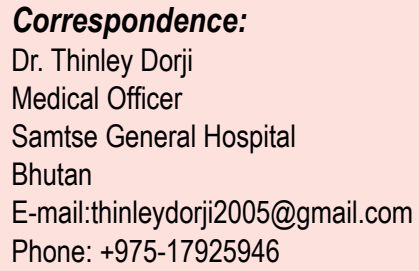

drug sensitive $T B$, spreads via respiratory aerosols and poses a risk for outbreaks ${ }^{4}$. MDR-TB can occur either due to infection with drug resistant strain (known as primary MDR-TB) or develop in those patients who were previously treated (known as secondary MDR-TB) ${ }^{3}$.

Globally in 2017, there were an estimated 0.558 million cases of MDR/RR-TB with $82 \%$ of them MDR-TB. Of these, $3.5 \%$ were among the new cases and $18 \%$ among the previously treated TB. ${ }^{1}$ Moreover of the total MDR-TB cases, $8.5 \%$ of them developed extensively drug resistant TB cases. Almost half of the cases of MDR-TB were from the three countries of India, China and Russia ${ }^{1}$. South East Asia region accounted for one third of global burden of MDR-TB in 2015. ${ }^{5}$ In Bhutan, the incidence rate of MDR/RR-TB was 22 per 100,000 population in $2017 .{ }^{4}$ Of this, $13 \%$ were among the new cases and $33 \%$ among retreated cases. ${ }^{18}$

In Bhutan, the National Tuberculosis Control Programme (NTCP) under the Ministry of Health is responsible for implementation of TB related preventive and promotive health activities. It initially started with short course chemotherapy followed by 
implementation of DOTS in $1997 .{ }^{6}$ The objectives of NTCP has been to increase the case notification and treatment success rate above $90 \%$ among prevalent TB cases and improve the MDR TB case detection and success rate above $75 \% .^{7}$ Bhutan has also ratified the sustainable development goals (SDG) of reducing the TB deaths by $90 \%$ and TB incidence by $80 \%$ compared to 2015 by $2030 .{ }^{8}$

Until 2010, there was no culture and drug sensitivity testing for TB in Bhutan. Patients were treated as a suspected case of MDR-TB based on clinical judgement. Since then, Lowenstein Jensen(LJ) medium has been used for the diagnosis of MDR-TB. This was followed by introduction of Mycobacteria Growth Indicator Tube (MGIT)in 2012 and line probe assay in 2014. Due to increasing number of MDR/RR cases, the GeneXpert testing has introduced in three Regional Hospitals and two hospitals of Phuntsholing and Samdrup Jongkhar since $2017^{9}$ and in Samtse General Hospital in 2019.

The treatment success of MDR-TB remains low at $55 \%$ compared to $82 \%$ in drug sensitive TB $^{1}$. The MDR-TB requires a treatment for at least 24 months while drug sensitive TB is treated for 6-8 months. In addition, while the drug sensitive TB costs around USD 2000 for the full course of treatment, MDR-TB requires almost 25 times that of drug sensitive TB. ${ }^{10}$

Although, the number of MDR-TB cases has been increasing in Bhutan, there are no published studies to access the predictors of MDR-TB at the hospital level. The objective of this study is to assess the prevalence, type of drug resistant TB and predictors of MDR-TB. The findings from this study will help to determine strategies to be taken in high burden areas like Samtse.

\section{METHODS}

\section{Study setting}

Samtse District is one of the most populated district located 275 kilometres from the capital Thimphu bordering the Indian State of West Bengal. Samtse district has a population of 62590 as of 2017. It has 2 hospitals and 2 Basic Health Unit (BHU) Grade 1 and 10 BHUs. The Samtse General Hospital serves as the referral point for all hospitals and $\mathrm{BHU}$ due to the availability of specialist care and diagnostic facilities. Samtse General Hospital is a 40 bedded district hospital with TB wards constructed exclusively for tuberculosis patients. Samtse has one of the highest tuberculosis burden in Bhutan. ${ }^{8}$

All patients who present to the hospital with clinical signs suggestive of TB (cough for more than two weeks with fever, chest pain, haemoptysis and weight loss) undergo sputum examination for TB by Ziehl-Neelsen stain and a chest $x$-ray. One early morning sputum sample and two spot sputum samples are collected in a sterile uricol container. Once the patient is diagnosed to have smear positive TB, they are admitted in the TB ward to break the chain of transmission of the disease.

The newly diagnosed patients on category one are admitted till they become smear negative while those on category two treatment (treatment failure and relapse) are kept for two months. All the TB patients who are admitted or transferred in from other health centres are given treatment card and a copy is retained at the hospital for future references. The TB card has patient information on age, sex, type of TB, treatment regime, weight and outcome of the treatment.

\section{Drug sensitivity testing}

The smear positive sputum samples are tested for drug resistance by GeneXpert MTB/RIF in the hospital. The sample processing and testing are performed as per the Standard Operating Procedures (SOP) of the Global Laboratory Initiative of the Stop TB Partnership. ${ }^{11}$ An additional sputum sample is shipped in cold chain to the Royal Centre for Disease Control (RCDC, National reference laboratory) for culture and drug testing using Lowenstein Jensen (LJ) medium. The reports are updated on TBISS (Tuberculosis Information and Surveillance System) which is a web based recording and reporting of TB.

\section{Study design and data collection}

This was aretrospectivecross sectional study conducted using the data extracted from the TB treatment cards maintained in the TB unit of the Samtse General Hospital. All the patients registered from $1^{\text {st }}$ January 2012 to $30^{\text {th }}$ June 2018 were included for the study. The information on age, sex, type of TB, treatment regime, weight and 
outcome of the treatment were obtained from the patents treatment card. Information on laboratory diagnosis were obtained from the TBISS website of RCDC.

\section{Ethical Clearance}

The ethical clearance was taken from the Research Ethical Clearance Board of Health (Ref. No.REBH/Approval/2018/037) under the Ministry of Health, Bhutan. Since this was a retrospective study with no direct contact with the patients, the informed consent was not found to be necessary. The names of the patients were coded for sake of confidentiality.

\section{Data management and analysis}

The data was entered and in IBM SPSS version 20 (SPSS Inc. Chicago. 2007) and analysed using STATA software. The data was double checked to ensure that they were no mistakes and discrepancy in data. Descriptive analysis was presented in frequency and percentages. The continuous variables like age and weight was converted into a categorical variable and compared using Chi square test and Fisher test to test if there is any associations with the dependent variable. Multiple logistic regressions with backward elimination were used to check for any associations between the variables. The $p$-value less than 0.05 were taken as the significant.

\section{RESULTS}

During the study period from January $1^{\text {st }} 2012$ to June 302018 , a total of 341 patients were registered in the TB unit of SamtseGeneral Hospital for the treatment. Of this $159(46.6 \%)$ were smear positive TB, $42(12.3 \%)$ were smear negative TB and $140(41.1 \%)$ were extra pulmonary tuberculosis (EPTB).

\section{Table 1: Characteristic of tuberculosis patients}

\begin{tabular}{|c|l|c|}
\hline \multirow{2}{*}{1} & Characteristic & $\mathrm{N}=159(\%)$ \\
\cline { 2 - 3 } & Sex & \\
\cline { 2 - 3 } & Male & $91(57.2)$ \\
\hline & Female & $68(42.8)$ \\
\hline \multirow{2}{*}{2} & Age group (years) & \\
\cline { 2 - 3 } & $<=14$ & $3(1.9)$ \\
\hline
\end{tabular}

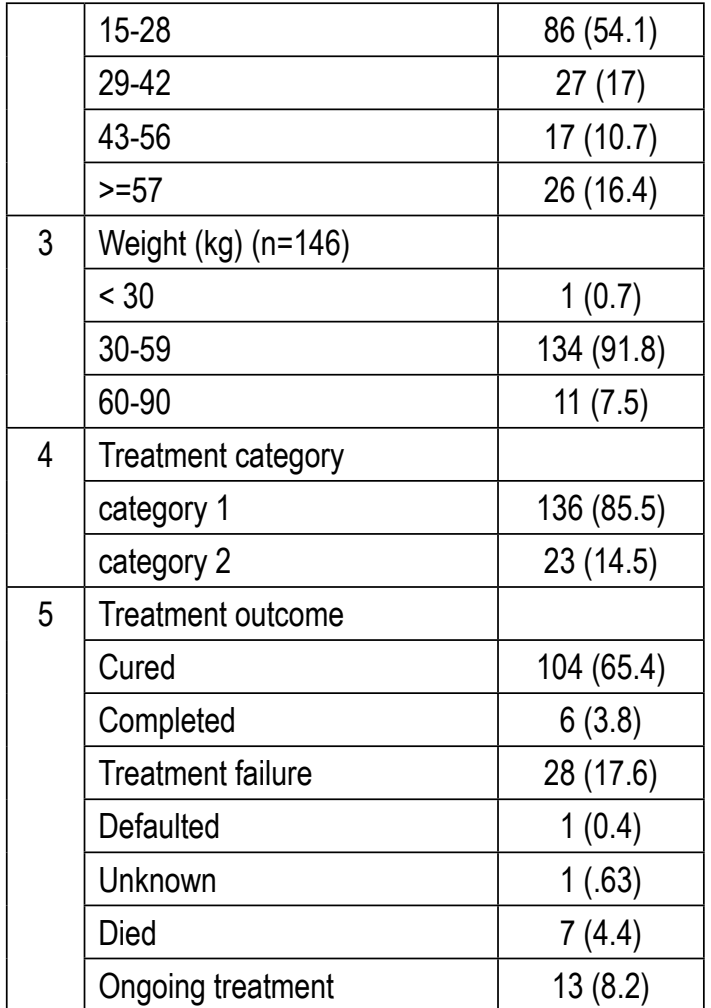

Out of 159 smear positive TB cases, 91 (57.2) were malepatients. Of total sputum positive cases, $104(65.41 \%)$ were declared cured, 6 (3.77\%) completed treatment and $13(8.18 \%)$ were still undergoing treatment at the time of study (Table 1). The majority of the cases $(71 \%)$ were seen in the age group between 15-42 years with mean age of 33.7 years. However, 28 patients (17.61\%) defaulted or had treatment failure and $7(4.4 \%)$ patients died while the status of one patient $(0.63 \%)$ was unknown.

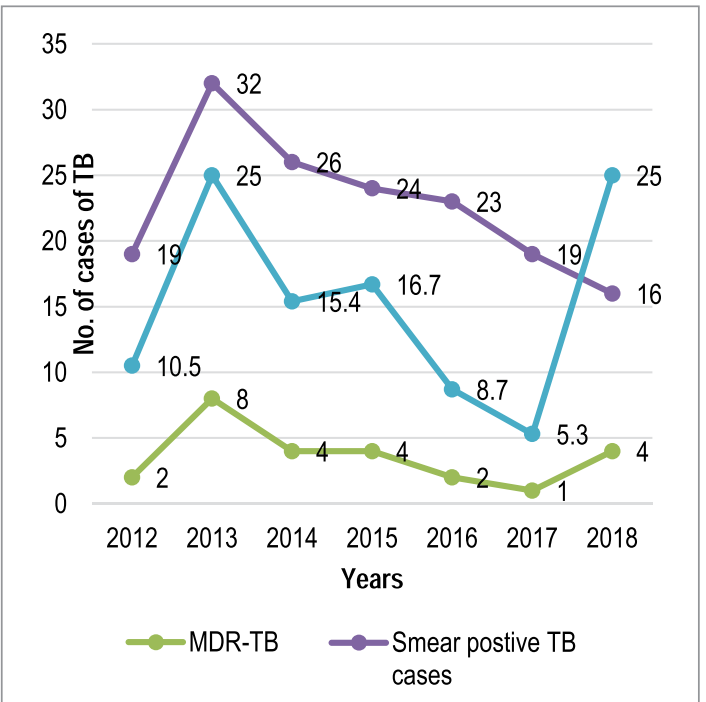




\section{Trend of MDR-TB}

Of the 159 patients, at least $33(21 \%)$ patients had resistant to at least one of the anti-tuberculosis drugs. The total number of MDR TB cases reported ranged from 1-8 cases with total of 25 cases in last six and half years (Figure 1). The annual prevalence of MDR-TB ranged from $5.3 \%$ to $25 \%$. The overall prevalence of MDR-TB was $16 \%$ (25 cases) out of 159 cases. The prevalence of MDR-TB among retreatment cases was $35 \%(n=8)$ while it was 13 $\%(n=17)$ among the new cases. During the same period, eight newly diagnosed tuberculosis patients developed mono-resistant to isoniazid.

\begin{tabular}{|c|c|c|c|c|}
\hline \multicolumn{5}{|c|}{ Table 2: Risk factors for MDR-TB patients } \\
\hline Age & $\begin{array}{l}\text { Non- } \\
\text { MDR }\end{array}$ & MDR & Total & $p$-value \\
\hline$<30$ years & \begin{tabular}{|l|}
72 \\
$(53.7 \%)$ \\
\end{tabular} & $\begin{array}{l}18 \\
(72 \%) \\
\end{array}$ & $\begin{array}{l}90 \\
(56.6 \%)\end{array}$ & \multirow[t]{2}{*}{0.124} \\
\hline$>30$ years & $\begin{array}{l}62 \\
(46.3 \%)\end{array}$ & $\begin{array}{l}7 \\
(28 \%)\end{array}$ & $\begin{array}{l}69 \\
(43.4 \%)\end{array}$ & \\
\hline \multicolumn{5}{|l|}{ Sex } \\
\hline Male & \begin{tabular}{|l|}
80 \\
$(59.7 \%)$ \\
\end{tabular} & \begin{tabular}{|l}
11 \\
$(44 \%)$ \\
\end{tabular} & $\begin{array}{l}91 \\
(57.2 \%) \\
\end{array}$ & 0.187 \\
\hline Female & $\begin{array}{l}54 \\
(40.3 \%) \\
\end{array}$ & $\begin{array}{l}14 \\
(56 \%) \\
\end{array}$ & \begin{tabular}{|l|}
68 \\
$(42.8 \%)$ \\
\end{tabular} & \\
\hline \multicolumn{5}{|c|}{ Weight $(n=195)$} \\
\hline$<50 \mathrm{~kg}$ & \begin{tabular}{|l|}
80 \\
$(59.7 \%)$ \\
\end{tabular} & $\begin{array}{l}15 \\
(60 \%) \\
\end{array}$ & $\begin{array}{l}95 \\
(59.8 \%) \\
\end{array}$ & \multirow[t]{2}{*}{1} \\
\hline$>=50 \mathrm{~kg}$ & $\begin{array}{l}54 \\
(40.3 \%) \\
\end{array}$ & $\begin{array}{l}10 \\
(40 \%) \\
\end{array}$ & \begin{tabular}{|l}
64 \\
$(40.3 \%)$ \\
\end{tabular} & \\
\hline \multicolumn{5}{|c|}{ Treatment category } \\
\hline category 1 & \begin{tabular}{|l|}
119 \\
$(88.8 \%)$ \\
\end{tabular} & \begin{tabular}{|l}
17 \\
$(68 \%)$ \\
\end{tabular} & \begin{tabular}{|l}
136 \\
$(85.5 \%)$
\end{tabular} & \multirow[t]{2}{*}{0.007} \\
\hline category 2 & $\begin{array}{l}15 \\
(11.2 \%) \\
\end{array}$ & \begin{tabular}{|l}
8 \\
$(32 \%)$ \\
\end{tabular} & $\begin{array}{l}23 \\
(14.5 \%) \\
\end{array}$ & \\
\hline
\end{tabular}

\section{Factors associated with MDR-TB}

There was a significant difference in MDR-TB among those on category 1 and category 2 TB patients ( $p$-value $=0.007$ ) (Table 2). While male sex suffered more from pulmonary TB, females constituted for $56 \%$ of total MDR-TB cases. However, there was no significant differences between male and female ( $p$-value $=0.187$ ).

During multiple regression, patients with previous treatments were significantly associated with MDRTB with adjusted odds ratio $4.59(95 \% \mathrm{Cl} .03-18)$ adjusting for the age. The patients under the age of 30 years were also 2.7 times more likely to develop MDR-TB (95\% Cl 1.01- 7.42) (Table 3).
Table 3: Adjusted Odds ratio for risk factors for MDRTB

\begin{tabular}{|l|l|l|l|}
\hline Characteristic & AOR & $p$-value & $95 \% \mathrm{Cl}$ \\
\hline Age $<30$ years & 2.7 & 0.046 & $1.01-7.42$ \\
\hline $\begin{array}{l}\text { Category } 2 \\
\text { treatment }\end{array}$ & 4.59 & 0.005 & $.03-.18$ \\
\hline
\end{tabular}

\section{DISCUSSION AND CONCLUSION}

In this study, the mean prevalence rate of MDRTB over last six and half years was $16 \%$. The prevalence of primary MDR-TB was $13 \%$ while secondary MDR-TB was 35\%. The correlates for MDR-TB in Samtse were age less than 30 years and patients in category 2 .

There are reports on the national prevalence of MDR-TB in Bhutan but no data at the hospital level. The prevalence of primary MDR-TB and secondary MDR-TB in this study is comparable to the national prevalence, where primary MDR-TBwas $13 \%$ and secondary MDR-TBat $33 \%$ in 2017. ${ }^{1}$ The burden of primary MDR-TB and secondary MDR-TB is higher in Bhutan than other South East Asia countries (Primary MDR-TB at 2.7\% and secondary MDR-TB at $13 \%$ )and globally (Primary MDR-TB $(3.5 \%)$ and secondary MDR-TB (18\%) ). ${ }^{1}$ It is not known why Bhutan has high prevalence rate of MDR-TB. This calls for further investigation in development of MDR-TB in Bhutan.However, Bhutan is expected to see increased detection of MDR-TB cases due to recent introduction of GeneXpert in all regional hospital and three district hospitals.

Patients previously treated for TB is strongly associated with MDR-TB. This has been seen in other studies done in other countries. ${ }^{12-16}$ Drug resistance is known to occur due to inappropriate use of anti-tuberculosis drugs. ${ }^{2}$ Younger people are more likely to develop drug resistant TB. ${ }^{2}$ Young being more active members of the community, increases their contact with MDR-TB patients thus increasing the probability of acquiring $\mathrm{TB}^{14-17}$ as well as their reluctance and irregular intake of drugs. ${ }^{13}$

It is a concern to see an increase in the number of MDR-TB cases in Bhutan as MDR-TB is associated with low treatment success, higher drug reactions and is comparatively more expensive. Multiple factors are responsible for development of MDR-TB like poor drug adherence, low income, young age, 
alcohol consumption, low educational status. ${ }^{17,19}$ The treatment of MDR-TB is usually associated with poorer outcome (global treatment success rate for MDR-TB is $54 \%$ compared to $83 \%$ in drug sensitive TB), more drug toxicity, longer duration of treatment and more financial burden compared to drug sensitive tuberculosis. ${ }^{20}$ The increase in MDR-TB could also lead to development of extremely drug resistant TB. The country status of MDR-TB cases indirectly shows the performance of the national tuberculosis control programme.

In Bhutan, the drug sensitivity for extra-pulmonary tuberculosis is not done on routine basis. Studies from India showed the drug resistant among extra pulmonary patients to be as high as $19 \% .{ }^{21}$ The increase in number of primary MDR-TB is one of the concern for tuberculosis program as it shows that transmission of MDR-TB is taking place in the community. Although, the diagnosed MDR-TB patients are admitted and treated in the hospitals to break the chain of transmission, there are still undiagnosed cases in the community which serves as the sources of transmission. So, active surveillance and further research needs to be undertaken to explore the means for development of primary MDR-TB in Bhutan.

\section{LIMITATIONS OF THE STUDY}

This study has several limitations to be considered. The study population is not representative of TB patients in Bhutan as it involved only one district hospital. Due to the retrospective nature of the study, patients risk behaviours like alcohol intake, smoking and drug adherence which are the risk factors for MDR-TB were not available

\section{CONFLICT OF INTEREST}

None

\section{REFERENCES}

1. World health Organization. Global Tuberculosis Report 2018.

2. Nachega JB, Chaisson RE. Tuberculosis drug resistance: a global threat. Clinical Infectious Diseases. 2003;36(Supplement_1):S24-S30.

3. World Health Organization. DRUG-RESISTANT TB SURVEILLANCE \& RESPONSE. 2014.
4. World Health Organization. THE SHORTER MDRTB REGIMEN. 2016.

5. Basnyat B, Caws M, Udwadia Z. Tuberculosis in South Asia: a tide in the affairs of men. Multidisciplinary respiratory medicine. 2018;13(1):10.

6. National Tuberculosis Control Programme. National guidelines for management of tuberculosis. 2016.

7. National Tuberculosis Control Programme. Program Profile of TB Control Program, CDD, DoPH Ministry of Health websiten.d [Available from: http://www.health.gov.bt/wp-content/uploads/ftps/ program-profiles/TB $\% 20$ control\%20program $\% 20$ profile.pdf.

8. National Tuberculosis Control Programme. National Strategic Plan to End TB 2017-2023. 2017.

9. Ministry of Health. Circular: GeneXpert facilities 2017 [Available from: http://www.health.gov.bt/ circular-3/.

10. Manjelievskaia J, Erck D, Piracha S, Schrager L. Drug-resistant TB: deadly, costly and in need of a vaccine. Transactions of the Royal Society of Tropical Medicine and Hygiene. 2016;110(3):18691.

11. Global Laboratory Initiative. Standard Operating Procedures (SOPs) 2019 [cited 2019. Available from: http://stoptb.org/wg/gli/sops.asp.

12. Dessalegn $M$, Daniel $E$, Behailu $S$, Wagnew $\mathrm{M}$, Nyagero J. Predictors of multidrug resistant tuberculosis among adult patients at Saint Peter Hospital Addis Ababa, Ethiopia. The Pan African Medical Journal. 2016;25(Suppl 2).

13. Workicho A, Kassahun W, Alemseged F. Risk factors for multidrug-resistant tuberculosis among tuberculosis patients: a case-control study. Infection and drug resistance. 2017;10:91.

14. Flora MS, Amin MN, Karim MR, Afroz S, Islam S, Alam A, et al. Risk factors of multi-drug-resistant tuberculosis in Bangladeshi population: a case control study. Bangladesh Medical Research Council Bulletin. 2013;39(1):34-41.

15. Ullah I, Javaid A, Tahir Z, Ullah O, Shah AA, Hasan $F$, et al. Pattern of drug resistance and risk factors associated with development of drug resistant Mycobacterium tuberculosis in Pakistan. PloS one. 2016;11(1):e0147529.

16. Rifat M, Milton AH, Hall J, Oldmeadow C, Islam MA, Husain A, et al. Development of multidrug resistant 
tuberculosis in Bangladesh: a case-control study on risk factors. PloS one. 2014;9(8):e105214.

17. Ahmad AM, Akhtar S, Hasan R, Khan JA, Hussain SF, Rizvi N. Risk factors for multidrug-resistant tuberculosis in urban Pakistan: a multicenter case-control study. International journal of mycobacteriology. 2012;1(3):137-42.

18. Law WS, Yew WW, Chiu Leung C, Kam KM, Tam $\mathrm{CM}$, Chan CK, et al. Risk factors for multidrugresistant tuberculosis in Hong Kong. The International Journal of Tuberculosis and Lung Disease. 2008;12(9):1065-70.
19. Mulu W, Mekkonnen D, Yimer M, Admassu A, Abera B. Risk factors for multidrug resistant tuberculosis patients in Amhara National Regional State. African health sciences. 2015;15(2):368-77.

20. World Health Organization. Global Tuberculosis Report, 2017. 2017 21/04/2018.

21. Dusthackeer A, Sekar G, Chidambaram S, Kumar V, Mehta P, Swaminathan S. Drug resistance among extrapulmonary TB patients: six years experience from a supranational reference laboratory. The Indian journal of medical research. 2015;142(5):568. 